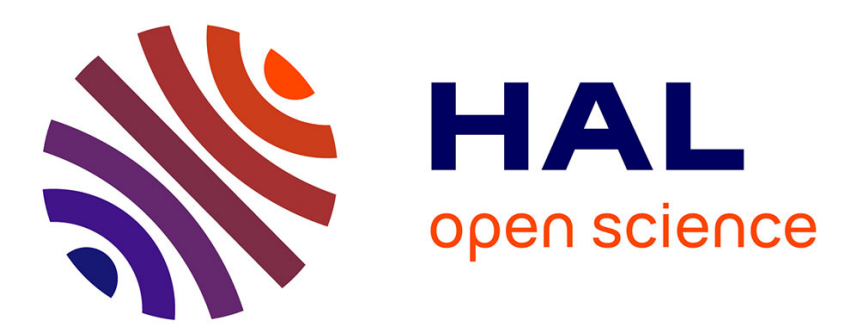

\title{
Displacement fields using correlation methods as a tool to investigate cell migration in 3D collagen gels
} Arnold Fertin, Laure Laforgue, Alain Duperray, Valérie M Laurent, Yves Usson, Claude Verdier

\section{- To cite this version:}

Arnold Fertin, Laure Laforgue, Alain Duperray, Valérie M Laurent, Yves Usson, et al.. Displacement fields using correlation methods as a tool to investigate cell migration in 3D collagen gels. Journal of Microscopy, 2019, 275 (3), pp.172-182. 10.1111/jmi.12825 . hal-02086505v2

\section{HAL Id: hal-02086505 \\ https://hal.science/hal-02086505v2}

Submitted on 24 Jun 2019

HAL is a multi-disciplinary open access archive for the deposit and dissemination of scientific research documents, whether they are published or not. The documents may come from teaching and research institutions in France or abroad, or from public or private research centers.
L'archive ouverte pluridisciplinaire HAL, est destinée au dépôt et à la diffusion de documents scientifiques de niveau recherche, publiés ou non, émanant des établissements d'enseignement et de recherche français ou étrangers, des laboratoires publics ou privés. 


\title{
Displacement fields using correlation methods as a tool to investigate cell migration in 3D collagen gels
}

\author{
Arnold Fertin $^{1 *}$ | Laure Laforgue ${ }^{2,3}$ | Alain Duperray ${ }^{2}$ \\ | Valérie M. Laurent ${ }^{3}$ | Yves Usson ${ }^{1 *}$ | Claude \\ Verdier $^{3 *}$
}

${ }^{1}$ Univ. Grenoble-Alpes, CNRS,

TIMC-IMAG, 38000 Grenoble, France

${ }^{2}$ Institute for Advanced Biosciences, INSERM U 1209, CNRS UMR 5309, Univ. Grenoble Alpes, 38000 Grenoble, France

${ }^{3}$ Univ. Grenoble-Alpes, CNRS, LIPhy, 38000 Grenoble, France

\section{Correspondence}

Arnold Fertin, Yves Usson, TIMC-IMAG, 38000 Grenoble, France

Email: arnold.fertin@univ-grenoble-alpes.fr, yves.usson@univ-grenoble-alpes.fr, claude.verdier@univ-grenoble-alpes.fr

Funding information

ANR grant No. 12-BS09-020-01.

LabeX Tec21 (No. ANR-11-LABX-0030).
Living cells embedded in a complex Extra-Cellular Matrix (ECM) migrate in a sophisticated way thanks to adhesions to matrix fibres and contractility. It is important to know what kind of forces are exerted by the cells. Here we use reflectance confocal microscopy to locate fibres accurately and determine displacement fields. Correlation techniques are used to this aim, coupled with proper digital image processing. Benchmark tests validate the method in the case of shear and stretching motions. Finally, the method is tested successfully for studying cancer cells migrating in collagen gels of different concentration.

\section{KEYWORDS}

Displacement field, phase correlation, confocal microscopy, cell migration, fibres, cancer cells.

\section{1 | INTRODUCTION}

Cell migration is a widely studied subject, requiring precise microscopic observations coupled with complementary biological experiments. In the past ten years, many processes related to cell migration have been considered, in particular on 2D functionalized gels [36] where cells can migrate. The understanding of the mechanisms of migration require an accurate knowledge of the spatio-temporal organization of actin fibres [39] and focal adhesions [29] within 
the cell. These mechanisms are highly dependent on molecular signaling, like the coordinated role of GTPases [21]. After a lamellipodium is formed at the front as well as focal adhesions, contractility is necessary for cells to detach their rear in order to move forward [36]. These phenomena seem easier to understand in 2D but remain difficult to investigate in $3 \mathrm{D}$ as the cells are embedded in the substrate and they might not have enough space to move. A lot of attention has been paid to realistic systems, in particular collagen, fibrin gels or matrigels within which cells can adhere and migrate. The early works of Friedl et al. [12] showed the ability of confocal reflectance microscopy techniques [11] to investigate cell migration in complex collagen networks. The method allows visualisation of fibre bundles or even individual fibres. This enables to propose 3D global mechanisms, close to the ones observed in 2D, with an extra step due to possible matrix degradation by MMPs (Matrix-MetalloProteases). Thus cells in 3D push a lamellipodium, form new adhesions, contract and bring their uropod. In addition, MMPs become necessary in dense collagen networks, or in a confined environment [42],

Another important aspect is the mechanics of cells: indeed cells forming focal adhesions on matrix fibres propel themselves by exerting forces which control their migration. It is possible that such forces correspond to the signature of particular types of cells. Thus it is fundamental to study the displacement fields generated around the cells, then determine forces using inverse methods already developed for elastic gels in 2D. Different methods have been proposed: the Boundary Integral Method (BIM, [7]), Traction Reconstruction from Point Force (TRPF, [35]), Fourier Transform Traction Cytometry (FTTC, [6]) or the Adjoint Method (AM, [3, 27]). These methods all need proper regularization and this question has been addressed in a relevant paper [32]. In three dimensions, experimental displacements or strain fields are usually harder to determine but have been obtained on regular grids [17, 13]. A relative small number of studies considering the inverse problem to obtain stress fields have been proposed in 3D [20,40,37]. The inverse problem is harder to solve as precise cell contour determination is needed since the elastic problem is now solved in the gel without the cell. In addition, the Green function $\mathbf{G}(\mathbf{r})$ linking displacements $\mathbf{u}(\mathbf{r})$ to stresses $\mathbf{T}(\mathbf{r})$ through $\mathbf{u}(\mathbf{r})=\int \mathbf{G}\left(\mathbf{r}-\mathbf{r}^{\prime}\right) \mathbf{T}\left(\mathbf{r}^{\prime}\right) \mathbf{d r}$ is not known (r being the space variable). To reach this goal, displacement fields with a very good resolution are needed, this being sometimes difficult due to the lower resolution of confocal microscopes in the Z-direction. In addition, the inverse problem needs an accurate displacement field close to the cell [40]. Therefore, one needs to develop specific correlation techniques, to link the fibres position after one time step. Such methods, also known as Digital Image Correlation (DIC) have been developed for many years in the fields of solid mechanics [31], fluid mechanics (Particle Image Velocimetry or PIV [5, 33]), geophysics [34], and more recently in biophysics $[25,16]$.

In this work, we propose to develop a new method based on images acquired on a confocal microscope using the reflectance technique, showing fluorescent cells (GFP-labeled cancer cells) in a collagen matrix, naturally reflecting fluorescence. Collagen fibres form bundles which can be easily detected and favour the use of the correlation technique. Specific tools are used to draw the cell contour first, in order to remove it from the collagen signal. This is achieved using cell segmentation [8]. Then the fibres signal is processed using phase correlation tools (POC) [2] with a high-accuracy subpixel image registration [38,4], taking advantage of the stronger intensities. After additional image processing is made, we test the method on benchmark problems, corresponding to shear, rotations and elongations of the network. Finally we apply the method to cancer cells migrating in different collagen networks - from low to high concentrations - and compare the displacement fields as well as the mechanisms used by these cells to migrate in this complex environment. 


\section{2 | MATERIALS AND METHODS}

\section{1 | Cell culture}

The cell line J82 was purchased from ATCC (HTB-1, ATCC Rockville, MD). This cell line was isolated from invasive bladder transitional cell carcinoma [23]. Cells were cultured at $37^{\circ} \mathrm{C}$ in a humidified $5 \% \mathrm{CO}_{2}$ atmosphere, in RPMI 1640 medium (Gibco, Saint Aubin, France) supplemented with 10\% fetal bovine serum (FBS), $100 \mathrm{UI} / \mathrm{mL}$ penicillin and $100 \mu \mathrm{g} / \mathrm{mL}$ streptomycin (complete RPMI medium). The cells were stably transfected with a plasmid expressing lifeact-GFP to stain F-actin [28], and these GFP-transfected cells were used for all experiments.

\section{2 | Collagen gel preparation and cell seeding}

Collagen gels prepared from rat tail collagen type I solutions (BD Biosciences) were cast in Nunc LabTek II chambered coverglass 8 wells. In order for the collagen gel to strongly adhere to the bottom glass surface of the wells, LabTeks were treated using the following protocol slightly modified from [41]: after a brief treatment (1 min) with sodium hydroxide $(\mathrm{NaOH} 0.1 \mathrm{~N})$, the LabTek was allowed to dry. 3-Aminopropyltrimethoxysilane (APTMS) was then added for $10 \mathrm{~min}$. After removing the chemical, the LabTek was cleaned 5 times with distilled water and dried. Finally, glutaraldehyde ( $0.5 \%$ in PBS) was added for $30 \mathrm{~min}$, the solution was removed, the Labtek was cleaned 5 times with distilled water and allowed to dry before adding the collagen solution. All the following preparation was carried out in ice to prevent polymerization of the collagen before adding it in the Labteks. The collagen solution consisted of RPMI medium supplemented with glutamine, FBS and antibiotics - at the same concentration as in the culture medium in which the cells were grown $-10 \mu \mathrm{g} / \mathrm{mL}$ of fibronectin, and three different concentrations of collagen $(0.95,1.8$ and $4.5 \mathrm{mg} / \mathrm{mL}$ ). After addition of $\mathrm{NaOH}(0.1 \mathrm{M})$ to reach a $\mathrm{pH}$ of 7.4 , freshly harvested $\mathrm{J} 82$ cells were included into the collagen mixture to obtain a final cell density of 2,000 cells $/ \mu \mathrm{L}$. This solution was inserted into the LabTeks and transferred to an incubator at $37^{\circ} \mathrm{C}$ for $30 \mathrm{~min}$ for the gel to polymerize. Fresh RPMI complete medium was added onto the gel after polymerization in order to make sure the cells would not lack nutrients during the experiment. Overall, the time between preparation of the gel and the beginning of the experiment was one hour.

\section{3 | Image acquisition and cell segmentation}

The structure of the collagen gels was visualized using confocal reflection microscopy. Image stacks of the gel were acquired on a Zeiss LSM 710 confocal microscope (Carl Zeiss, Jena, Germany) as previously described [15]. Collagen fibres were visualized, with a 40x/1.1NA water immersion objective (Zeiss) and imaged at a wavelength of $633 \mathrm{~nm}$. Imaging of fluorescently labeled actin was conducted with the same objective, at a wavelength of $488 \mathrm{~nm}$. During the experiments, samples were placed in a temperature and $\mathrm{CO}_{2}$ controlled on-stage incubator $\left(37^{\circ} \mathrm{C}, 5 \% \mathrm{CO}_{2}\right)$. By denoising with a Gaussian filter, and thresholding the intensities of the GFP-fluorescence (green channel), the cell volume was defined. From this segmentation [8], the cell volume was removed from the red channel. As a consequence, the collagen signal was left without the cell, since this is the relevant information to use. In addition, only the most intense levels were kept. Fig. 1(A) shows the red channel with both cell and collagen whereas Fig. 1(B) shows the green channel allowing to identify the cell contour. 


\section{3 | DISPLACEMENT FIELD ALGORITHM}

\section{1 | Definitions}

In this work, we propose to use the Phase Only-Correlation (POC) function to estimate the displacement field between two volumes $V_{1}$ and $V_{2}$ at two consecutive time steps $t_{1}$ and $t_{2}$. Typically volume sizes are chosen to be $512 \times 512 \times N_{z}$ ( $N_{z}$ is the number of slices in the range [30; 100]). Indeed, during typical elapsed time (10 min), cells have moved within the filamentous gel and have displaced the fibres. These fibres are used as tracers to determine displacements. Thus once they are localized at time $t_{1}$ using a maximum intensity criterion, it is easier to locate them at time $t_{2}$ using the POC function.

Let us first define vectors to be used in the phase correlation method and subvoxel estimation. We need to use voxel position vectors $\boldsymbol{x}$ with components $\left(x_{1}, x_{2}, x_{3}\right)$ and volume fluorescence intensity $V(\boldsymbol{x}, t)$ at position $\boldsymbol{x}$ and time $t$. The volume intensity $V_{t_{1}}(\boldsymbol{x})=V\left(\boldsymbol{x}, t_{1}\right)$ at time $t_{1}$ will be deformed to $V_{t_{2}}(\boldsymbol{x})=V\left(\boldsymbol{x}, t_{2}\right)$ at time $t_{2}$.

We define a discrete set $E=\left\{\boldsymbol{x}^{(i)} ; i=1, \ldots, M\right\}$ with $M$ positions, at which we estimate the local displacements $\boldsymbol{u}^{(i)}$. We define this set $E$ from the initial volume $V_{t_{1}}$ by selecting $\boldsymbol{x}^{(i)}$ voxels corresponding to local fluorescence peaks, i.e. local three-dimensional maxima above a threshold given by the well-known Otsu method [26]. This ensures a selection of positions located on collagen fibres with a high local signal to noise ratio.

To estimate the local displacement between $t_{1}$ and $t_{2}$ at a given position $\boldsymbol{x}^{(i)}$, we define two sub-volumes $v_{t_{1}}^{(i)}$ and $v_{t_{2}}^{(i)}$ centered on this position, and perform discrete calculations:

$$
\begin{gathered}
v_{t}^{(i)}(\boldsymbol{x})=a(\boldsymbol{x}) V_{t}\left(x_{1}+x_{1}^{(i)}-\frac{N_{1}}{2}, x_{2}+x_{2}^{(i)}-\frac{N_{2}}{2}, x_{3}+x_{3}^{(i)}-\frac{N_{3}}{2}\right) \\
x_{1} \in\left\{0, \ldots N_{1}-1\right\}, x_{2} \in\left\{0, \ldots N_{2}-1\right\}, x_{3} \in\left\{0, \ldots N_{3}-1\right\},
\end{gathered}
$$

with $\left(N_{1}, N_{2}, N_{3}\right)$ the sub-volume sizes (i.e. width, height and depth, denoted later by $\left.N_{1} \times N_{2} \times N_{3}\right)$, and $a(x)$ the weight defined by the type of window function (e.g. Hamming, Blackman etc...) [14]. $\left(N_{1}, N_{2}, N_{3}\right)$ are chosen as powers of two, since Discrete Fourier Transform algorithms are usually optimized for such signal sizes.

We will now determine the displacement $\boldsymbol{u}^{(i)}$ which will be estimated in two steps : first we calculate the discrete part $\boldsymbol{u}_{d}^{(i)}$, and then the sub-voxel estimation $\boldsymbol{\delta} \boldsymbol{u}^{(i)}$. Finally, we will find $\boldsymbol{u}^{(i)}=\boldsymbol{u}_{d}^{(i)}+\boldsymbol{\delta} \boldsymbol{u}^{(i)}$ (see Figure 2).

\section{2 | First step : discrete estimation of displacement field}

The phase correlation method is a well known frequency domain method to relate two signals. In order to estimate the shift between $v_{t_{1}}^{(i)}(\boldsymbol{x})$ and $v_{t_{2}}^{(i)}(\boldsymbol{x})$, we calculate their normalized cross-power spectrum:

$$
R^{(i)}(\boldsymbol{\omega})=\frac{\overline{v_{1}^{(i)}(\boldsymbol{\omega})} v_{2}^{(i)}(\boldsymbol{\omega})}{\left|\overline{v_{1}^{(i)}(\boldsymbol{\omega})} v_{2}^{(i)}(\boldsymbol{\omega})\right|}, v_{1}^{(i)}(\boldsymbol{\omega})=\mathscr{F}\left(v_{t_{1}}^{(i)}(\boldsymbol{x})\right), v_{2}^{(i)}(\boldsymbol{\omega})=\mathscr{F}\left(v_{t_{2}}^{(i)}(\boldsymbol{x})\right)
$$

where $\mathscr{F}$ denotes Fourier transform and $\overline{v_{1}^{(i)}(\boldsymbol{\omega})}$ denotes the complex conjugate of $v_{1}^{(i)}(\boldsymbol{\omega})$. The inverse Fourier transform of $R^{(i)}(\boldsymbol{\omega})$ gives the so called Phase-Only Correlation (POC) function:

$$
r^{(i)}(\boldsymbol{x})=\mathscr{F}^{-1}\left(R^{(i)}(\boldsymbol{\omega})\right)
$$


The most noticeable property of the POC function is that it exhibits a unique sharp peak when the signal $v_{t_{2}}^{(i)}$ is a shifted version of $v_{t_{1}}^{(i)}$. Using this property, we obtain the required displacement $\boldsymbol{u}_{d}^{(i)}$ in voxels, by locating the position of the peak of $r(\boldsymbol{x})^{(i)}$ :

$$
\boldsymbol{u}_{d}^{(i)}=\underset{\boldsymbol{x}}{\arg \max }\left(r^{(i)}(\boldsymbol{x})\right)
$$

An example of the peak determination from the normalized cross-correlation $r^{(i)}(\boldsymbol{x})$ - or POC - is shown in the following Figure 3, where two typical images at times $t_{1}$ and $t_{2}$ are shown, and the POC, in the $\left(x_{1}, x_{2}\right)$ coordinate system, shows a clear maximum, indicating the coordinates of the displacement vector.

\subsection{Second step : sub-voxel estimation of displacement field}

The size of sub-volumes in the previous step are chosen large enough to estimate the expected value of $\boldsymbol{u}_{d}$, but in practice the maximum of correlation and peak location precision obtained from equation (4) decrease if voxels in subvolumes are submitted to various shifts $[22,18]$. Therefore we first started with a large window and obtained the estimate $\boldsymbol{u}_{d}^{(i)}$ of the displacement using phase correlation as described above, where displacements are expressed in voxels. Then a smaller box was used to estimate the extra displacement, as shown previouvsly. The sub-pixel resolution as explained by Foroosh [9] was found quite appropriate to calculate $\delta \boldsymbol{u}^{(i)}$. Basically, the method corrects the value of the displacement in voxels, by adding/subtracting a number corresponding to the ratio of POC values of the peak and its neighbors. This is sketched in Fig. 2. The final displacement $\boldsymbol{u}^{(i)}$ is the sum of the first one $\boldsymbol{u}_{d}^{(i)}$ and the second one, $\delta \boldsymbol{u}^{(i)}$ obtained during the second step, $\boldsymbol{u}^{(i)}=\boldsymbol{u}_{d}^{(i)}+\delta \boldsymbol{u}^{(i)}$.

Note that this method is insensitive to the type of images that are used. We only use the most intense levels (as explained in §2.3), underlying the structure of the objects. So these objects can be of different kinds, as long as the fluorescence levels are well defined. Any other sub-structures can be treated, not necessary filaments.

\section{EXPERIMENTAL RESULTS}

\subsection{Displacements and errors}

In order to estimate the method accuracy, we have created nine data-sets from real volume acquisition at three different collagen concentrations : $0.95 \mathrm{mg} / \mathrm{mL}, 1.8 \mathrm{mg} / \mathrm{mL}$ and $4.5 \mathrm{mg} / \mathrm{mL}$ (three data sets per concentration). From these acquisitions we created in silico experiments, i.e. we built synthetic volumes by applying numerically known deformations to real collagen images and interpolated with quintic splines to reduce numerical errors [24]. Each data set is formed by the real raw volume (first time step $t_{1}$ ) and its deformed version (second time step $t_{2}$ ).

The theoretical displacement $U(\boldsymbol{x})$ is given by the following expressions in shear and uni-axial elongation.

Shear case : $U_{1}(\boldsymbol{x})=\gamma x_{2} ; U_{2}(\boldsymbol{x})=0 ; U_{3}(\boldsymbol{x})=0$.

Elongation case : $U_{1}(\boldsymbol{x})=\epsilon x_{1} ; U_{2}(\boldsymbol{x})=-\frac{\epsilon}{2} x_{2} ; U_{3}(\boldsymbol{x})=-\frac{\epsilon}{2} x_{3}$.

where $\gamma$ is the shear strain and $\epsilon$ the elongation. Note that the displacement $U(\boldsymbol{x})$ is calculated in terms of $\boldsymbol{x}$ corresponding to the position of the fibres in the undeformed volume. Then we use our algorithm on the deformed network to verify whether the method is efficient for the determination of the displacements. The errors $E$ are given by the square of the norm of the difference between calculated and expected displacements $E=\|u-U\|^{2}$. Values of $\gamma$ and $\epsilon$ are chosen so that displacements do not exceed 16 voxels. Generally we used three concentrations with 
three cases (i.e. three collagen gels) for each concentration, leading to nine data sets. Fig. 4(A) shows the theoretical displacement field corresponding to a mixed combination of shear and elongation using $\gamma=0.039$ and $\epsilon=0.031$. The result of the algorithm is shown in Fig. 4(B), corresponding to reconstruction of the displacements. Relative errors are given w.r.t. to the voxel size in Figs 4(C-D). Here we used the voxel diagonal as a measure of its size.

Finally, results of another test case, called the 'dipole', are presented in Fig.5. The idea was here to be close to a real situation where a cell applies more localized forces, for example during migration. This could correspond to two rapidly decaying opposed displacements localized at points $\mathrm{A}\left(x_{1}^{A}, x_{2}^{A}, x_{3}^{A}\right)$ and $\mathrm{B}\left(x_{1}^{B}, x_{2}^{B}, x_{3}^{B}\right)$. The displacement field is given by $\boldsymbol{U}(\boldsymbol{x})=\boldsymbol{U}_{0} \exp \left(-K\left|\boldsymbol{r}^{A}\right|^{2}\right)-\boldsymbol{U}_{0} \exp \left(-K\left|\boldsymbol{r}^{B}\right|^{2}\right)$, with $\left|\boldsymbol{r}^{A}\right|=\sqrt{\left(x_{1}-x_{1}^{A}\right)^{2}+\left(x_{2}-x_{2}^{A}\right)^{2}+\left(x_{3}-x_{3}^{A}\right)^{2}}$, and $\left|\boldsymbol{r}^{B}\right|=\sqrt{\left(x_{1}-x_{1}^{B}\right)^{2}+\left(x_{2}-x_{2}^{B}\right)^{2}+\left(x_{3}-x_{3}^{B}\right)^{2}}$. $U_{0}$ is a displacement vector pointing from $\mathrm{A}$ to $\mathrm{B}$, and $\mathrm{K}$ is a constant that can be adjusted to obtain a large strain, in order to test the method robustness. We find that the method allows good reconstruction of the imposed displacements, even for a large value of $K$, when the decrease is sharp, leading to large strains. Fig. 5 also represents a real cell, with the chosen end points A and B, where displacements are localized. For this case, relative errors are also presented in Fig. 6.

\section{2 | Parameter optimisation}

Different parameters were tested in what follows. In particular, calculation of the displacement field can lead to a first approximation using a large initial window. Generally, it is better to consider a second pass, leading to a better approximation of displacements. Then, as shown in Fig. 2, one reiterates this procedure to get a better accuracy. The second window should be smaller in order to avoid edge effects, while the initial one was larger to account for larger displacements. The influence of window sizes has been tested in Fig. 4(C). The first graph shows the role of window dimensions $(32 \times 32 \times 16$ vs. $64 \times 64 \times 16)$ in voxels on the relative error, given as the percentage of voxel diagonal. One can see that using a larger window size for a first pass works better, since displacements can be large. The optimal window is $32 \times 32 \times 16$ in this case. In Fig. $4(\mathrm{C})$, we also show the influence of the second pass using different window sizes $(4 \times 4 \times 4,8 \times 8 \times 8,16 \times 16 \times 16$ and $64 \times 64 \times 16)$. The relative error is minimum for the size $16 \times 16 \times 16$. Obviously the smaller size $4 \times 4 \times 4$ leads to numerical errors in the Fourier transform. Increasing this size leads a better accuracy (i.e. smaller errors), but as window size increases, edge effects appear again so one finds a best compromise using window size $16 \times 16 \times 16$ for the second pass. All results (except for the size $4 \times 4 \times 4$ ) show that improved accuracy is obtained for two passes (i.e. sub-voxel resolution), as compared to one only. Finally, the influence of window filtering is tested in Fig. 4(D) and shows very similar values of the relative error (in terms of voxel size again) whatever the window function. Blackman, Cosine, Hamming, Hann, Tukey and Welch windowing effects were tested and give similar low relative errors, whereas a rectangular window is less accurate. In what follows, the Hamming window will be used.

Next, the influence of different mechanical deformations has been tested, in particular a shear, an elongation, a combined shear/stretch, and finally a displacement dipole (i.e. two opposed local displacements). The distinct calculation of relative errors in $\mathrm{XY}$ and Z-planes show the influence of the confocal resolution which is poor in the Z-direction due to various reasons already discussed [10]. But the relative errors found here remain rather small. In the dipole case, the relative errors are larger, due to the higher deformation in the Z-direction, whereas the other cases mostly involve deformations in the XY-plane. This can be seen in Fig. 6(A). Note that the mean Z-error is $0.113 \pm 0.088 \mu \mathrm{m}$, which is larger than the mean $\mathrm{XY}$-error $(0.061 \pm 0.028 \mu \mathrm{m})$. When plotted in terms of relative error with respect to voxel size $(0.277 \mu \mathrm{m}$ for $\mathrm{XY}$ and $0.77 \mu \mathrm{m}$ for $\mathrm{Z})$, the relative error is slightly higher for the $\mathrm{Z}$-direction. To summarize, the errors remain small.

In addition, Fig. 6(B) shows the effect of noise. The noise was chosen as a proportion of the input image variance. Noise only gives rise to large errors when it becomes quite large (greater than $50 \%$ ). This demonstrates the ability of 
our technique to describe accurately the proper fibre deformations.

\section{3 | Displacement field de-noising}

To reduce the effect of noise, two additional steps to improve the computation of the displacement field $\boldsymbol{u}(\boldsymbol{x})$ were validated. First a test on outliers was used in order to eliminate those measurements that are irrelevant. This was achieved by collecting all the displacements measured and making use of a threshold criterion to eliminate data cor-

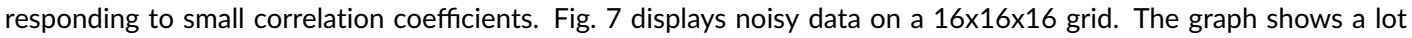
of uncorrelated data and a smaller number of data points exhibit high correlation. The threshold used here is 8.7, meaning a percentile of $1 \%$. This value will be used in what follows, in particular in Figures 8(A-B).

The second step is to get rid of more unrelevant data, such as the ones left in Fig. 8(B), with wrong direction. A few uncorrelated vectors still remain that will be discarded. For this purpose, the local coherence of displacement vector directions $\left(u_{1}, u_{2}, u_{3}\right)$ was used as a selection criterion. Using the nearest neighbors within a given sphere (a radius of $10 \mu \mathrm{m}$ was chosen), we compared the current components with those of the neighbors. If the direction of one of the components was found out of the range $\left[Q_{1}-1.5 * I Q R ; Q_{3}+1.5 * I Q R\right]$ (see definitions in Fig. 8), we simply removed the vector. This does not eliminate so many vectors in the end, as seen in Fig. 8(C), where it is clear that the most relevant data remains.

\subsection{Application to a real biological case : cancer cell migration in 3D collagen gel}

In order to apply the method to a particular biological experiment, we chose to use three collagen concentrations. Cancer cells of invasive type (J82 epithelial bladder cancer cell line) were seeded in collagen gels at three different concentrations $0.95 \mathrm{mg} / \mathrm{mL}, 1.8 \mathrm{mg} / \mathrm{mL}$ and $4.5 \mathrm{mg} / \mathrm{mL}$. The behavior of such cells was followed in time and confocal images were acquired in order to exhibit the motion of cells in such media. The method described above was applied to exhibit displacement fields, in particular in the vicinity of J82 cells. An example of such displacement fields is shown in Fig. 9(A-B-C) for the three collagen concentrations (0.95 mg/mL, $1.8 \mathrm{mg} / \mathrm{mL}$ and $4.5 \mathrm{mg} / \mathrm{mL})$. One can see clearly the decrease of the norm of the displacement (see color code) when collagen concentration is increasing. Values range from $4.5 \mu \mathrm{m}$ down to $1.6 \mu \mathrm{m}$ respectively from the low concentration to the high concentration. This is relevant as we expect cells to displace low concentration collagen fibres more easily, since the network is looser. Further details will be discussed in what follows.

\section{5 | DISCUSSION AND CONCLUSIONS}

The main motivation of the current work was to construct a robust method enabling to determine accurate displacement fields in the context of cell migration within soft matrices, in particular collagen or biological gels for which information about fibres is known. This is indeed the case when using collagen fibres reflecting naturally incoming light from a laser [11, 15].

Recent works have been devoted to such analyses. In particular, Ravichandran and co-authors [10, 25] developed 3D digital volume correlation (DVC) on sub-volumes to determine full-field displacements with a very good accuracy $(0.2 \mu \mathrm{m})$ and low noise $(0.05-0.1 \mu \mathrm{m})$. This was achieved thanks to 3D confocal stacks on labeled fibrin gels, an extra step providing more contrast. They obtained sub-voxel accuracy with 3D quadratic polynomial fitting of the correlation function near the peak. Their method can be extended to large deformations using stretch correlation procedures 
[10]. The interesting idea of their work was to couple matrix deformations together with the matrix microstructural details, as also shown in our previous work [15].

In another study, Kim et al. [16] used 3D confocal microscopy stacks together with a partial volume correlation (PVC) method in two steps. FFT or direct-cross-correlation based particle image velocimetry were used on 2Dslices using sub-windows. Then normalized cross-correlation in terms of $\Delta Z$ shifts was used to find the maximum crossgrained Z-deformation, and sub-pixel resolution was obtained by fitting a gaussian around the maximum. We actually used this method initially but it was found less accurate than doing a full 3D POC.

The method developed here is rather simple and involves sub-pixel accuracy [2, 4, 9]. It uses the most intense levels, i.e. fluorescence peaks above a given level, giving rise to an unstructured mesh. The Phase-Only-Correlation (POC) is directly applied on 3D sub-volumes, and exhibits sharp peaks (Fig. 3). Two passes are used, one to catch the coarse displacement (large sub-window), and the second one (small sub-window) to attain sub-pixel resolution using combinations of neighboring peaks [9]. So the method is fast, provides good resolution and low relative errors.

This was checked in details using benchmark cases (shear, elongation, mixed shear/elongation and dipole) on numerically deformed collagen images. The first pass using the correlation algorithm needs a rather large box size (step 1 in Fig. 2, and relevant tests in Fig. 4), for example 32×32×16, depending on the experiment. This is a good compromise for accuracy. Indeed large displacements can be measured, and edge effects are not a problem. Then a second pass was used with smaller box size, for example $16 \times 16 \times 16$. Again this is an optimal solution for increasing accuracy, as boxes can reach the main corners, and this size is small enough to refine the initial displacement field. Benchmark solutions were shown to give good comparisons between theoretical and estimated displacements, in the case of shear (thus involving rotations), stretch, combined shear/stretch (Fig. 4(A-B)), and the case of the dipole (Fig. 5). Fig. 6(A) shows the good performance of the method on these various cases, both regarding the relative XYand Z-errors. Note that the Z-error is not as good but still quite reasonable, in the case of the dipole. The effect of noise has also been studied and reveals a robustness for reasonable values of the noise $(<50 \%)$.

This method could be applied to other types of fibres or sub-structures. It is general enough and applies to all voxels in the field of view, not only points located on a regular grid [31,17]. In particular this is important since we can use the locations where the fluorescence is higher. This has been shown to be quite relevant here. Fig. 1, for example, shows regions of space where very intense fibres are located, that can be used for better correlation estimations. Several Traction Force Microscopy methods (TFM) based on finite element methods [3, 27, 30] use this advantage because meshes can be made according to the nodes positions, therefore they do not need any further interpolation, like the FTTC method [6, 32]. In addition, the method allows the determination of large displacements as opposed to classical ones (PIV, etc.) requiring images to be close enough for adequate processing.

Application to cells in a collagen matrix gives quite interesting data. Accurate results are found everywhere even close to the cell, as can be seen from the spatial distribution of the maximum of correlation in Fig. 8 (based on the criterion in Fig. 7) and further refinements. High correlation is still found near the cell at distances of few microns. Finally, the displacement norms were shown to decrease with the increase of the collagen concentration. This makes sense, and further interesting local effects were observed. For the low concentration $(0.95 \mathrm{mg} / \mathrm{mL})$, strong displacements up to $4 \mu \mathrm{m}$ are found in the cell vicinity, indicating that cells are pulling on the collagen fibres. This effect is also observed for the medium concentration $(1.8 \mathrm{mg} / \mathrm{mL})$ in agreement with cell polarity, because displacements point to the rear of the cell. This motion seemed to correspond to mesenchymal migration [36]. Finally, lateral effects corresponding to symetric displacement of $\approx 1 \mu \mathrm{m}$ are found for the most concentrated gel $(4.5 \mathrm{mg} / \mathrm{mL})$. These effects possibly indicate a different mode of migration, the amoeboid type of motion [11], which is probably related to the fact that this cell is constrained by the gel. Note that displacements seem to be correlated with cell shapes : at low and medium concentrations cells are elongated, whereas at high concentration the cell looks round. 
Therefore not only we observe a clear effect of the gel concentration on the displacement of the cells (with inward displacements), but also we showed that the technique can be interesting to determine variations in the modes of migration. This opens up a new route for accurate determination of displacement fields, to be used in combination with adequate TFM tools [40] allowing to determine forces exerted by cells.

Further works are now needed to investigate the forces exerted by cells in 3D, and potential new effects might be found by comparing cells of different invasiveness. This new indicator might correspond to a cell signature, to be associated with cell invasiveness [19]. This new method may also reveal to be important for elucidating new modes of migration, in particular MMP-driven mechanisms where matrix degradation is important, that still remain to be understood [12, 42].

\section{Acknowledgements}

We thank Alexei Grichine for help with the microscopy facilities and the IBiSA Platform. This work was supported by ANR grant No. 12-BS09-020-01 (TRANSMIG). The team at LIPhy is a member of LabeX Tec21 (Investissements d'Avenir, grant agreement No. ANR-11-LABX-0030). We are grateful to Arnaud Chauvière for interesting suggestions and improvment of the manuscript.

\section{references}

[1] Ahrens J, Geveci B, Law C. Paraview: An end-user tool for large data visualization. The visualization handbook 2005;717.

[2] Alba A, Aguilar-Ponce RM, Vigueras-Gómez JF, Arce-Santana E. Phase correlation based image alignment with subpixel accuracy. In: Adv. Artificial Intell. Springer; 2012.p. 171-182.

[3] Ambrosi D, Duperray A, Peschetola V, Verdier C. Traction patterns of tumor cells. J Math Biol 2009;58:163-181.

[4] Balci M, Foroosh $\mathrm{H}$. Subpixel registration directly from the phase difference. EURASIP J Advances Signal Processing 2006;2006:1-12.

[5] Bastiaans RJ. Cross-correlation PIV; theory, implementation and accuracy. Eindhoven University of Technology, Faculty of Mechanical Engineering; 2000.

[6] Butler JP, Tolic-Nørrelykke IM, Fabry B, Fredberg JJ. Traction fields, moments, and strain energy that cells exert on their surroundings. Am J Physiol Cell Physiol 2002;282(3):C595-C605.

[7] Dembo M, Wang YL. Stresses at the cell-to-substrate interface during locomotion of fibroblasts. Biophys J 1999;76(4):2307-2316.

[8] Ersoy I, Bunyak F, Mackey MA, Palaniappan K. Cell segmentation using Hessian-based detection and contour evolution with directional derivatives. In: 15th IEEE Int. Conf. Image Process.; 2008. .

[9] Foroosh H, Zerubia JB, Berthod M. Extension of phase correlation to subpixel registration. IEEE Trans Image Process 2002;11(3):188-200.

[10] Franck C, Hong S, Maskarinec S, Tirrell D, Ravichandran G. Three-dimensional full-field measurements of large deformations in soft materials using confocal microscopy and digital volume correlation. Exp Mech 2007;47(3):427-438.

[11] Friedl P, Borgmann S, Bröcker EB. Amoeboid leukocyte crawling through extracellular matrix: lessons from the Dictyostelium paradigm of cell movement. J Leukoc Biol 2001;70(4):491-509.

[12] Friedl P, Wolf K. Proteolytic interstitial cell migration: a five-step process. Cancer Metastasis Rev 2009;28(1-2):129-135. 
[13] González-Avalos P, Mürnseer M, Deeg J, Bachmann A, Spatz J, Dooley S, et al. Quantification of substrate and cellular strains in stretchable 3D cell cultures: an experimental and computational framework. J Microscopy 2017;266:115-125.

[14] Harris FJ. On the use of windows for harmonic analysis with the discrete Fourier transform. Proceedings of the IEEE 1978;66(1):51-83.

[15] Iordan A, Duperray A, Gérard A, Grichine A, Verdier C. Breakdown of cell-collagen networks through collagen remodeling. Biorheology 2010;47:277-295.

[16] Kim J, Jones CAR, Groves NS, Sun B. Three-Dimensional Reflectance Traction Microscopy. PLoS One 2016;11(6):e0156797.

[17] Koch TM, Münster S, Bonakdar N, Butler JP, Fabry B. 3D Traction forces in cancer cell invasion. PLoS One 2012;7(3):e33476.

[18] Kuglin C. The phase correlation image alignment method. In: Proc. Int. Conf. on Cybernetics Society; 1975. p. $163-165$.

[19] Laforgue L. Migration de cellules cancéreuses dans des gels de collagène 3D. PhD thesis, Université Grenoble Alpes; 2016.

[20] Legant WR, Miller JS, Blakely BL, Cohen DM, Genin GM, Chen CS. Measurement of mechanical tractions exerted by cells in three-dimensional matrices. Nat Methods 2010;7(12):969-971.

[21] Machacek M, Hodgson L, Welch C, Elliott H, Pertz O, Nalbant P, et al. Coordination of Rho GTPase activities during cell protrusion. Nature 2009;461(7260):99-103.

[22] Manduchi R, Mian GA. Accuracy analysis for correlation-based image registration algorithms. In: 1993 IEEE Int. Symp. Circuits \& Systems; 1993. p. 834-837 vol.1.

[23] Masters JR, Hepburn PJ, Walker L, Highman WJ, Trejdosiewicz LK, Povey S, et al. Tissue culture model of transitional cell carcinoma: characterization of twenty-two human urothelial cell lines. Cancer Res 1986;46(7):3630-3636.

[24] Meijering EH, Niessen WJ, Viergever MA. Quantitative evaluation of convolution-based methods for medical image interpolation. Medical Image Analysis 2001;5(2):111-126.

[25] Notbohm J, Lesman A, Tirrell DA, Ravichandran G. Quantifying cell-induced matrix deformation in three dimensions based on imaging matrix fibers. Integr Biol 2015;7:1186-1195.

[26] Otsu N. A threshold selection method from gray-level histograms. IEEE Trans on systems, man, and cybernetics 1979;9(1):62-66.

[27] Peschetola V, Laurent VM, Duperray A, Michel R, Ambrosi D, Preziosi L, et al. Time-dependent traction force microscopy for cancer cells as a measure of invasiveness. Cytoskeleton 2013;70(4):201-214.

[28] Riedl J, Crevenna AH, Kessenbrock K, Yu JH, Neukirchen D, Bista M, et al. Lifeact: a versatile marker to visualize F-actin. Nat Methods 2008;5(7):605-607.

[29] Riveline D, Zamir E, Balaban NQ, Schwarz US, Ishizaki T, Narumiya S, et al. Focal contacts as mechanosensors: externally applied local mechanical force induces growth of focal contacts by an mDia1-dependent and ROCK-independent mechanism. J Cell Biol 2001;153(6):1175-1186.

[30] Roux C, Duperray A, Laurent VM, Michel R, Peschetola V, Verdier C, et al. Prediction of traction forces of motile cells. Interface Focus 2016;6:20160042.

[31] Roux S, Réthoré J, Hild F. Digital image correlation and fracture: an advanced technique for estimating stress intensity factors of 2D and 3D cracks. J Phys D: Appl Phys 2009;42:214004. 
[32] Sabass B, Gardel ML, Waterman CM, Schwarz US. High resolution traction force microscopy based on experimental and computational advances. Biophys J 2008;94(1):207-220.

[33] Scarano F. Iterative image deformation methods in PIV. Meas Sci Technol 2001;13(1):1.

[34] Schmedes J, Archuleta RJ, Lavallée D. Correlation of earthquake source parameters inferred from dynamic rupture simulations. J Geophys Res 2010;115:B03304.

[35] Schwarz US, Balaban NQ, Riveline D, Bershadsky A, Geiger B, Safran SA. Calculation of forces at focal adhesions from elastic substrate data: the effect of localized force and the need for regularization. Biophys J 2002;83(3):1380-1394.

[36] Sheetz MP, Felsenfeld D, Galbraith CG, Choquet D. Cell migration as a five-step cycle. Biochem Soc Symp 1999;65:233243.

[37] Steinwachs J, Metzner C, Skodzek K, Lang N, Thievessen I, Mark C, et al. Three-dimensional force microscopy of cells in biopolymer networks. Nat Methods 2016;13:171-176.

[38] Takita K, Sasaki Y, Higuchi T, Kobayashi K. High-accuracy subpixel image registration based on phase-only correlation. IEICE Trans on fundamentals of electronics, communications \& computer sciences 2003;86(8):1925-1934.

[39] Théry M, Pépin A, Dressaire E, Chen $Y$, Bornens $M$. Cell distribution of stress fibres in response to the geometry of the adhesive environment. Cell Motil Cytoskeleton 2006;63(6):341-355.

[40] Vitale G, Preziosi L, Ambrosi D. A numerical method for the inverse problem of cell traction in 3D. Inverse Problems 2012;28:095013.

[41] Wang YL, Pelham RJ. Preparation of a flexible, porous polyacrylamide substrate for mechanical studies of cultured cells. Methods Enzymol 1998;298:489-496.

[42] Wolf K, Lindert MT, Krause M, Alexander S, Riet JT, Willis AL, et al. Physical limits of cell migration: control by ECM space and nuclear deformation and tuning by proteolysis and traction force. J Cell Biol 2013;201(7):1069-1084. 


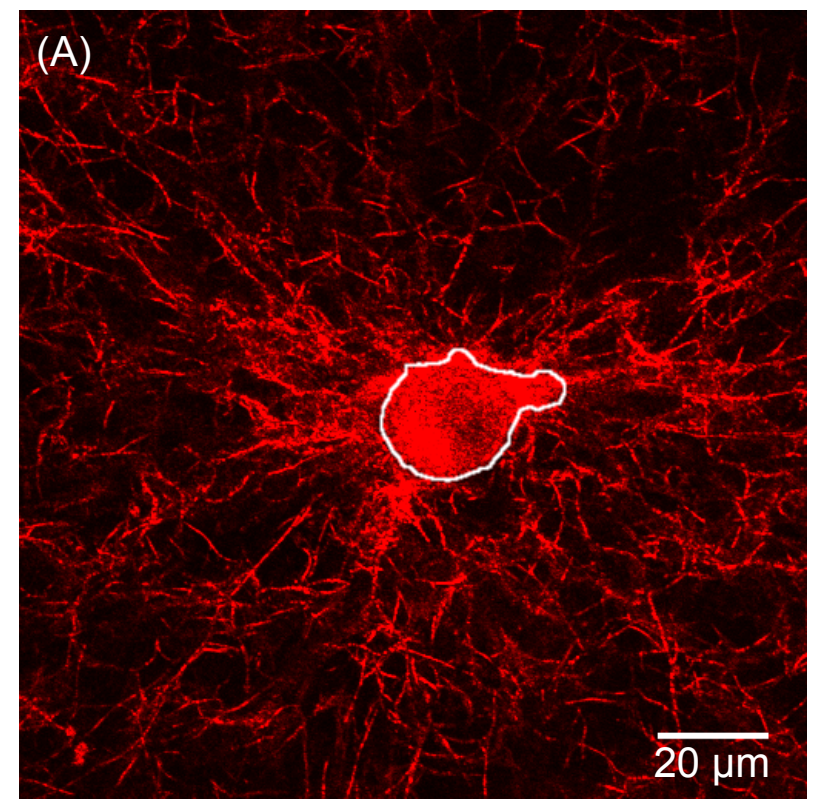

(B)

\section{$\overline{20 \mu m}$}

FIGURE 1 (A) Cell among collagen fibres (red channel), collagen concentration c=0.95mg $/ \mathrm{mL}$. (B) Fluorescent cell. In (A) and (B) the white line shows cell contour segmented from green channel. 


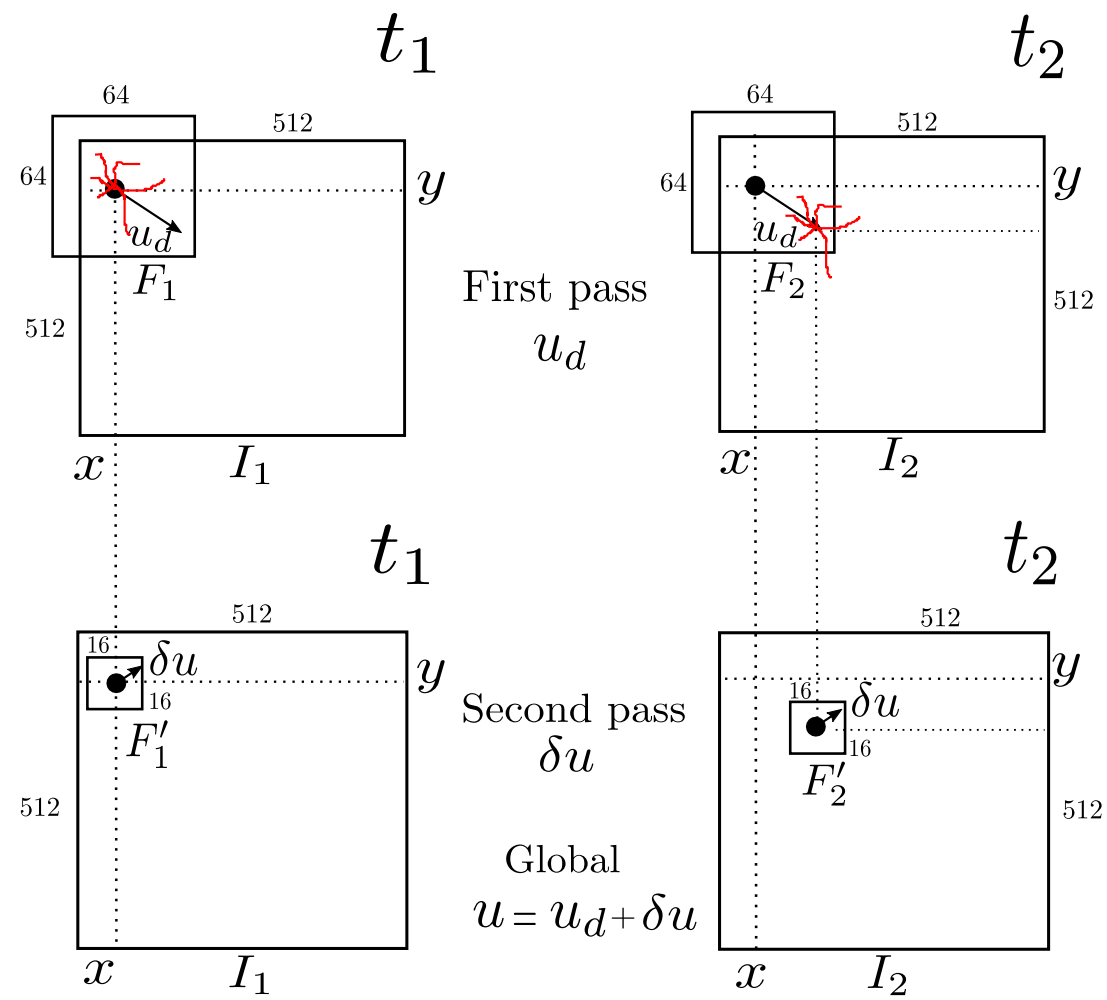

FIGURE 2 Box refinement method to increase displacement accuracy, sketched in 2D. In this drawing, an initial rough box $F_{1}$ of size $64 \times 64$ is chosen. A first correlation is made at position $(x, y)$ in order to obtain displacement $\boldsymbol{u}_{\boldsymbol{d}}(x, y)$, between $F_{1}$ (time $t_{1}$ ) and $F_{2}$ (time $t_{2}$ ), at pixel resolution. Then refinement is made using a smaller size box $\left(F_{1}^{\prime}\right)$, here $16 \times 16$ giving a better estimate $\delta \boldsymbol{u}(x, y)$, leading to the final displacement between $F_{1}^{\prime}\left(t_{1}\right)$ and $F_{2}^{\prime}\left(t_{2}\right)$, namely $\boldsymbol{u}(x, y)=\boldsymbol{u}_{\boldsymbol{d}}(x, y)+\boldsymbol{\delta} \boldsymbol{u}(x, y)$, with sub-pixel resolution. Remark: Box sizes are just for the sketch, and not on scale. 
A

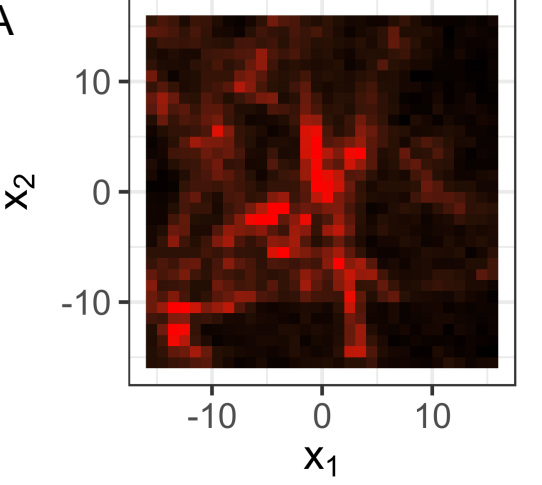

B

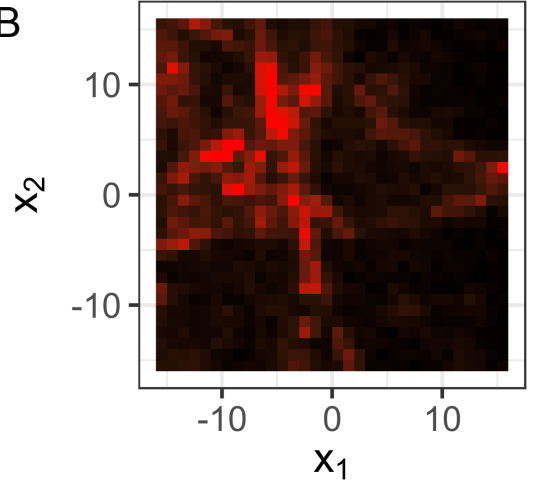

C

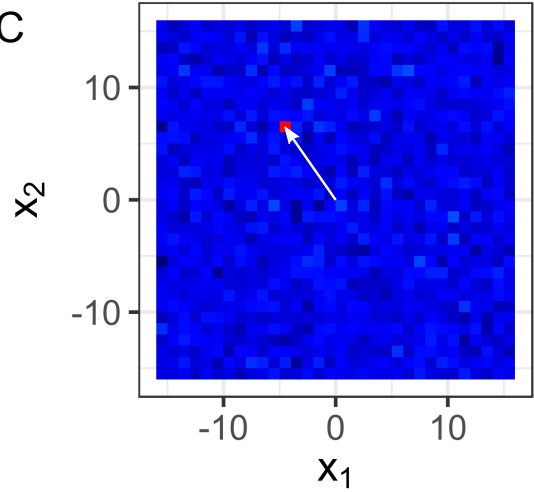

$\mathrm{v}_{\mathrm{t}_{1}}^{(\mathrm{i})}\left(\mathrm{x}_{1}, \mathrm{x}_{2}\right)$

$=250$

200

150

100

50 $\mathrm{v}_{\mathrm{t}_{2}}^{(\mathrm{i})}\left(\mathrm{x}_{1}, \mathrm{x}_{2}\right)$

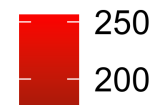

200

150

100

50

0 $r^{(i)}\left(x_{1}, x_{2}\right)$

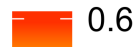

0.4

0.2

0.0

FIGURE 3 Bidimensional illustration of the POC function response. (A)-(B) Two images of collagen fibres at respective times $t_{1}$ and $t_{2}$. A known shift $\boldsymbol{u}_{d}=(-5,6)$ is applied to image (A) to produce image (B). (C) POC function or $r^{(i)}(\boldsymbol{x})$ in the $\left(x_{1}, x_{2}\right)$ plane, with peak location indicating the coordinates of the displacement vector, see white arrow. 
(A)

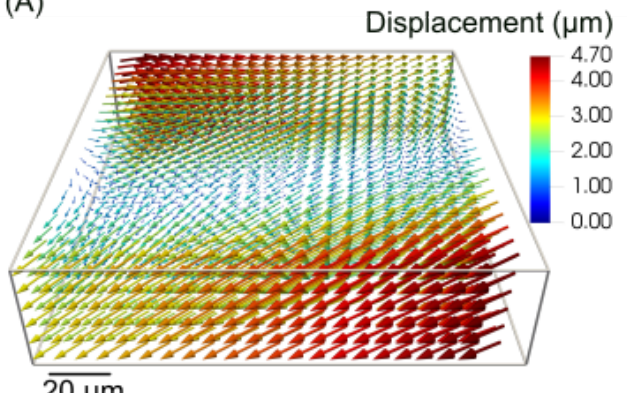

$$
\overline{20 \mu \mathrm{m}}
$$

(C)

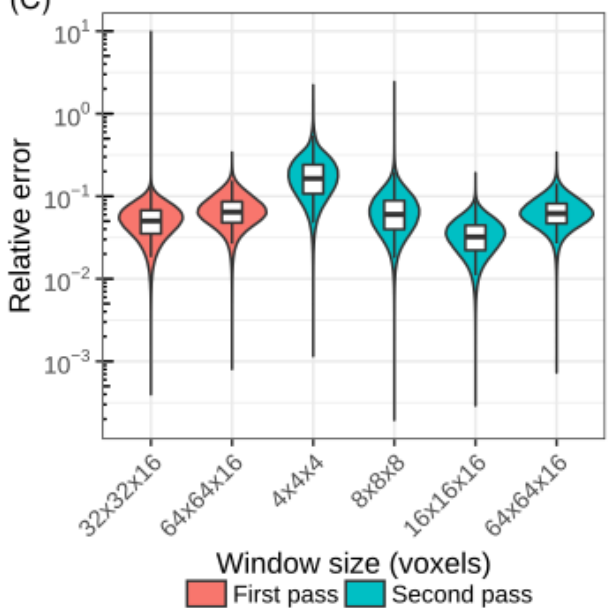

(B)

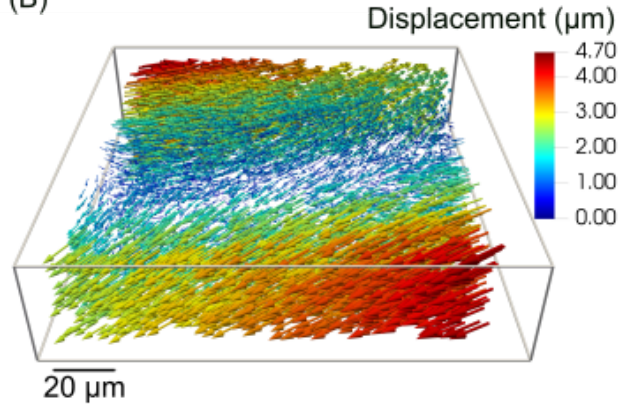

(D)

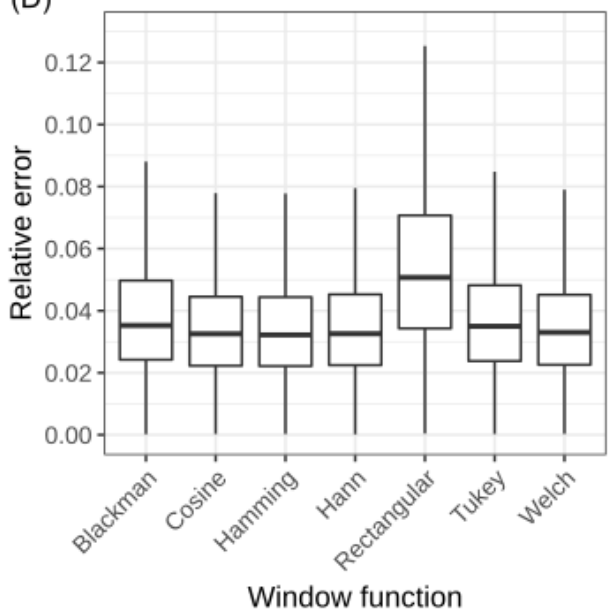

FIGURE 4 Shear combined with a stretch $(\gamma=0.039, \epsilon=0.031)$ (A) theoretical displacement, (B) calculated displacement with optimal parameters: first pass with a $64 \times 64 \times 16$ window and second pass $16 \times 16 \times 16$, both passes with a Hamming window function. (C) Relative error distributions (w.r.t. to voxel size) showing the superposition of boxplot and violin plot, with one pass or two passes, as a function of window size. Using too small windows

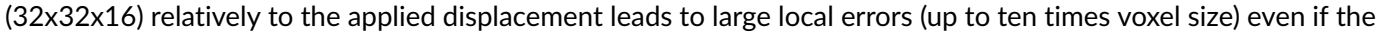
average relative errors remain small. Using a second pass can lead to a smaller relative error ( $16 \times 16 \times 16$ here). (D) Relative error distributions (w.r.t. voxel size) depending on windowing type. Window function does not affect the results much. 

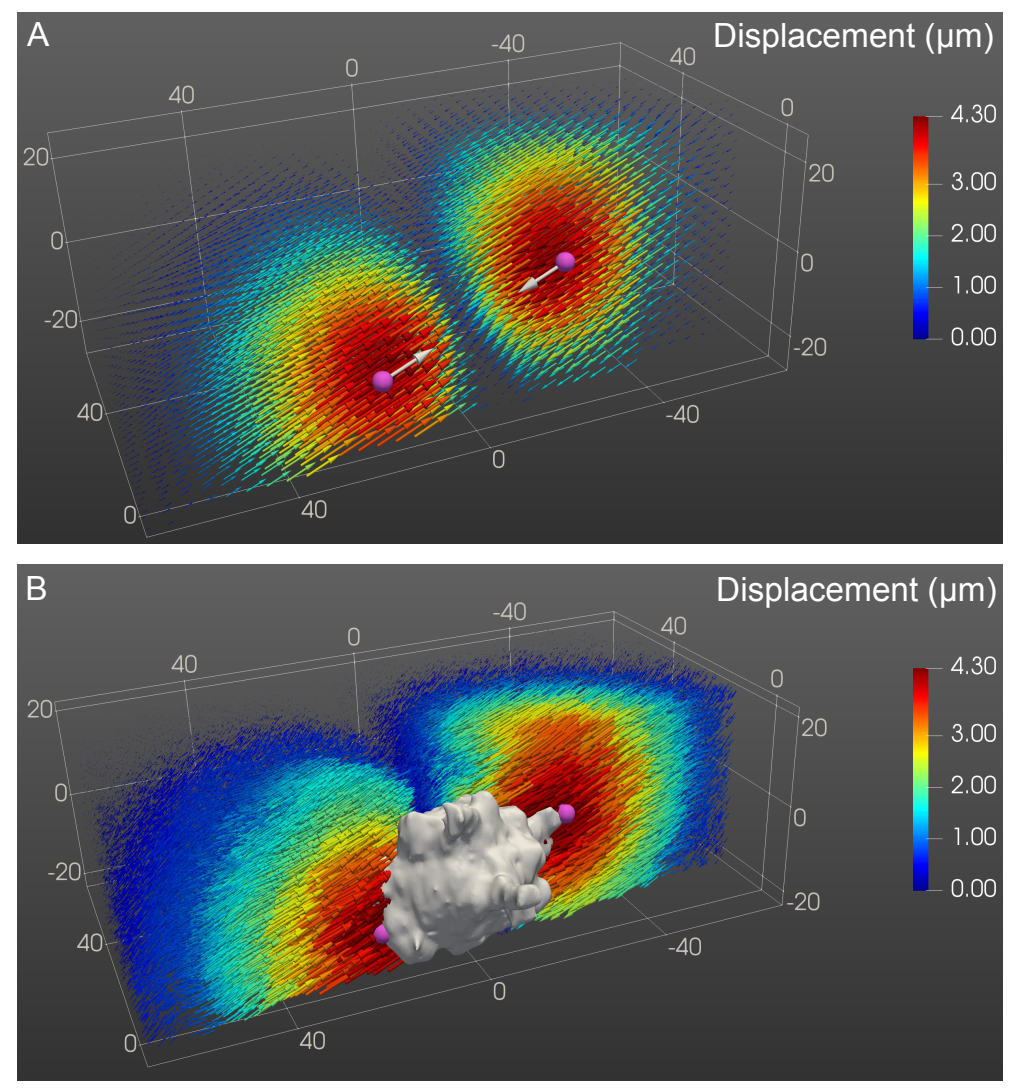

FIGURE 5 Dipole case. Prescribed deformations are used at two positions $\mathrm{A}\left(x_{1}^{A}, x_{2}^{A}, x_{3}^{A}\right)$ and $\mathrm{B}\left(x_{1}^{B}, x_{2}^{B}, x_{3}^{B}\right)$ with opposed directions, simulating a cell migrating within a matrix. The corresponding imposed deformation field is $\boldsymbol{U}(\boldsymbol{x})=\boldsymbol{U}_{0} \exp \left(-K\left|\boldsymbol{r}^{A}\right|^{2}\right)-\boldsymbol{U}_{0} \exp \left(-K\left|\boldsymbol{r}^{B}\right|^{2}\right)$, with $\mathrm{K}=0.001 \mu \mathrm{m}^{-2}$ and $\mathrm{U}_{0}=4.7 \mu \mathrm{m}$ (see definitions in $\S 4.1$ ). Comparison between prescribed displacements (A) and calculated ones using the POC (B). Note that the cell is also represented, with its ends $A$ and $B$ where the displacements are localized. 

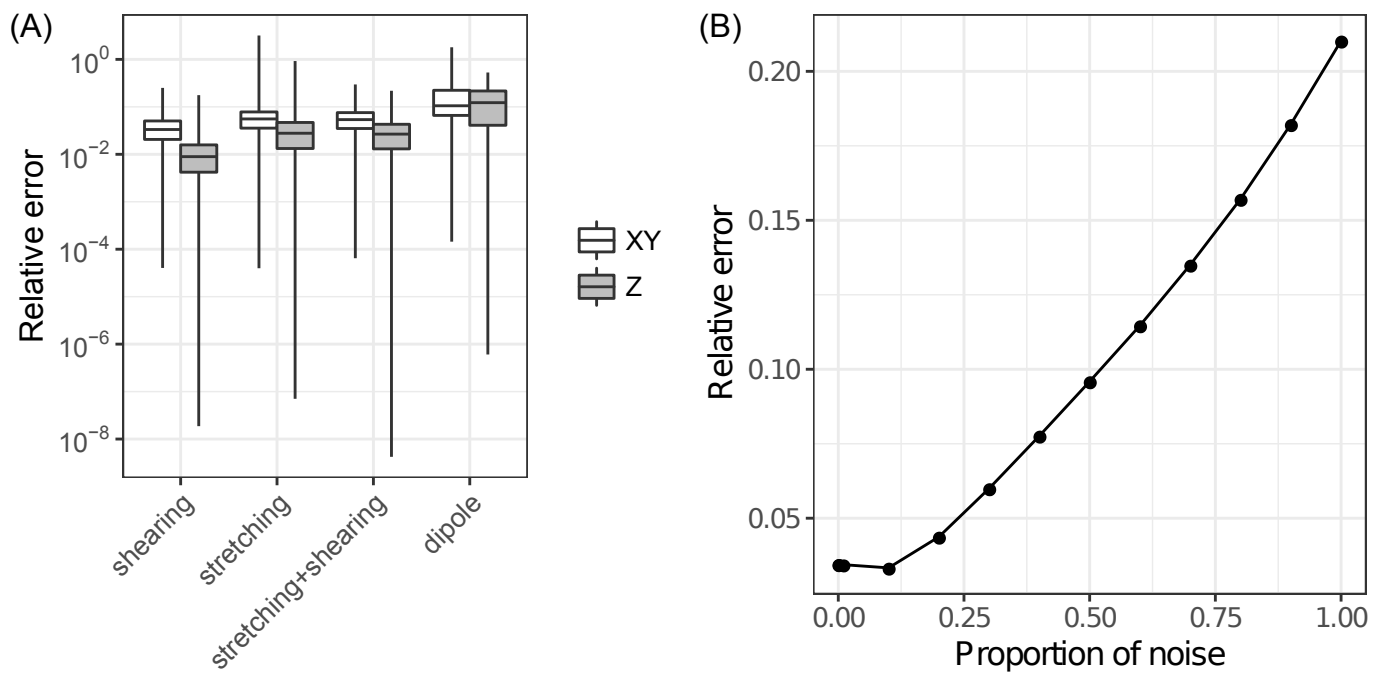

FIGURE 6 (A) Relative errors for four cases. Shearing ( $\gamma=0.0625)$, stretching $(\epsilon=0.0625)$, combined shearing and stretching experiments $(\gamma=0.039$ and $\epsilon=0.031)$ and 'dipole case' (from Fig.5). For each case the relative error is represented in the $\mathrm{XY}$ or Z-plane. Note that the relative errors in the Z-direction are relatively small, but slightly higher for the dipole case. (B) Effect of noise on relative errors.

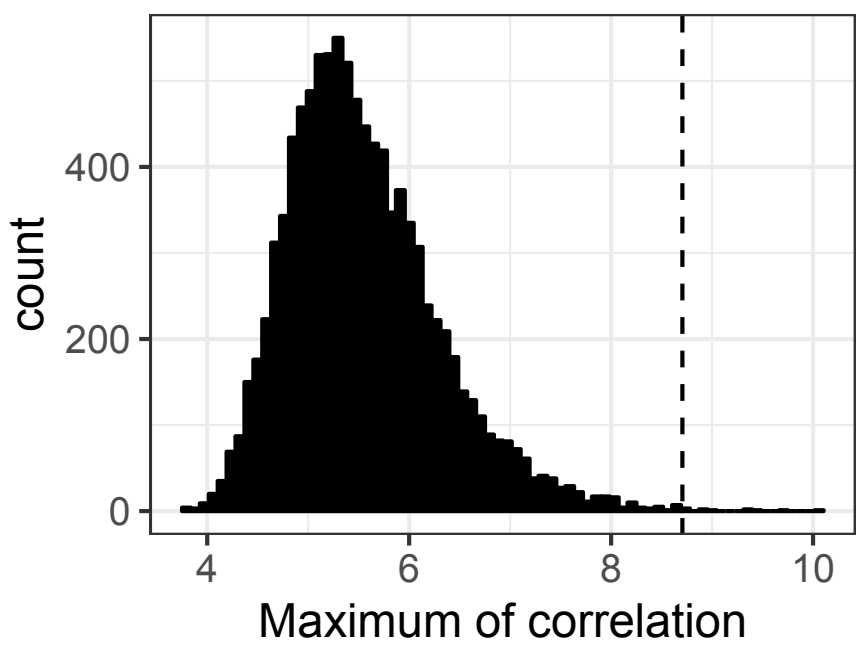

FIGURE 7 Maximum correlation for noisy data on a $16 \times 16 \times 16$ box. Most of the data (99\%) has a very small correlation coefficient whereas $1 \%$ of the data is above the threshold 8.7 , and has a high correlation coefficient. 

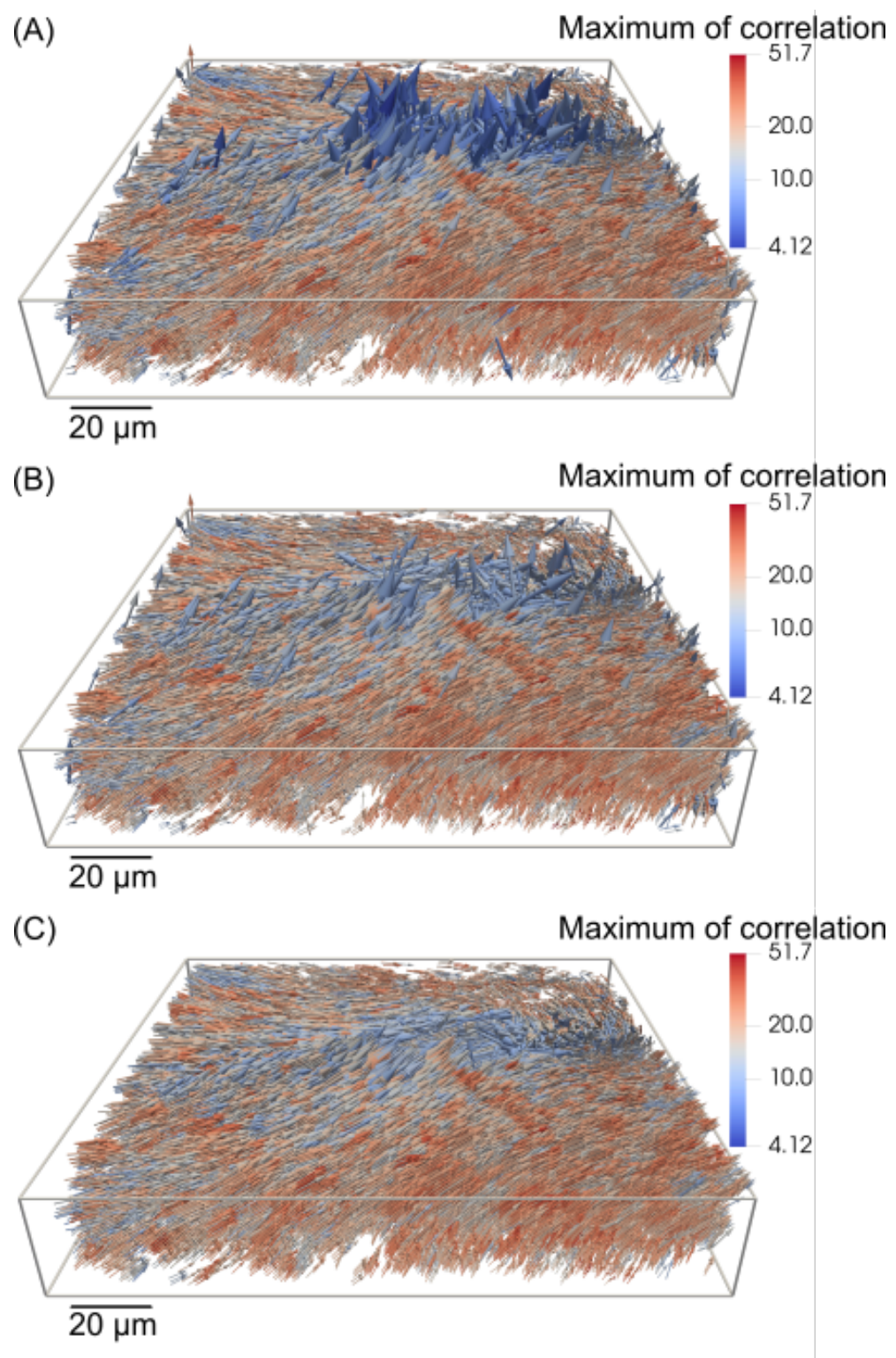

FIGURE 8 Maximum correlation for (A) raw data, (B) data following thresholding with value of 8.7 from Fig. 7 (C) data from (B) after removing displacement vectors with wrong direction not in range $\left[Q_{1}-1.5 * I Q R ; Q_{3}+1.5 * I Q R\right]$, using nearest neighbors to compute first and third quartiles $\left(Q 1\right.$ and $\left.Q_{3}\right)$ and interquartile range $I Q R=Q_{1}-Q_{3}$. 

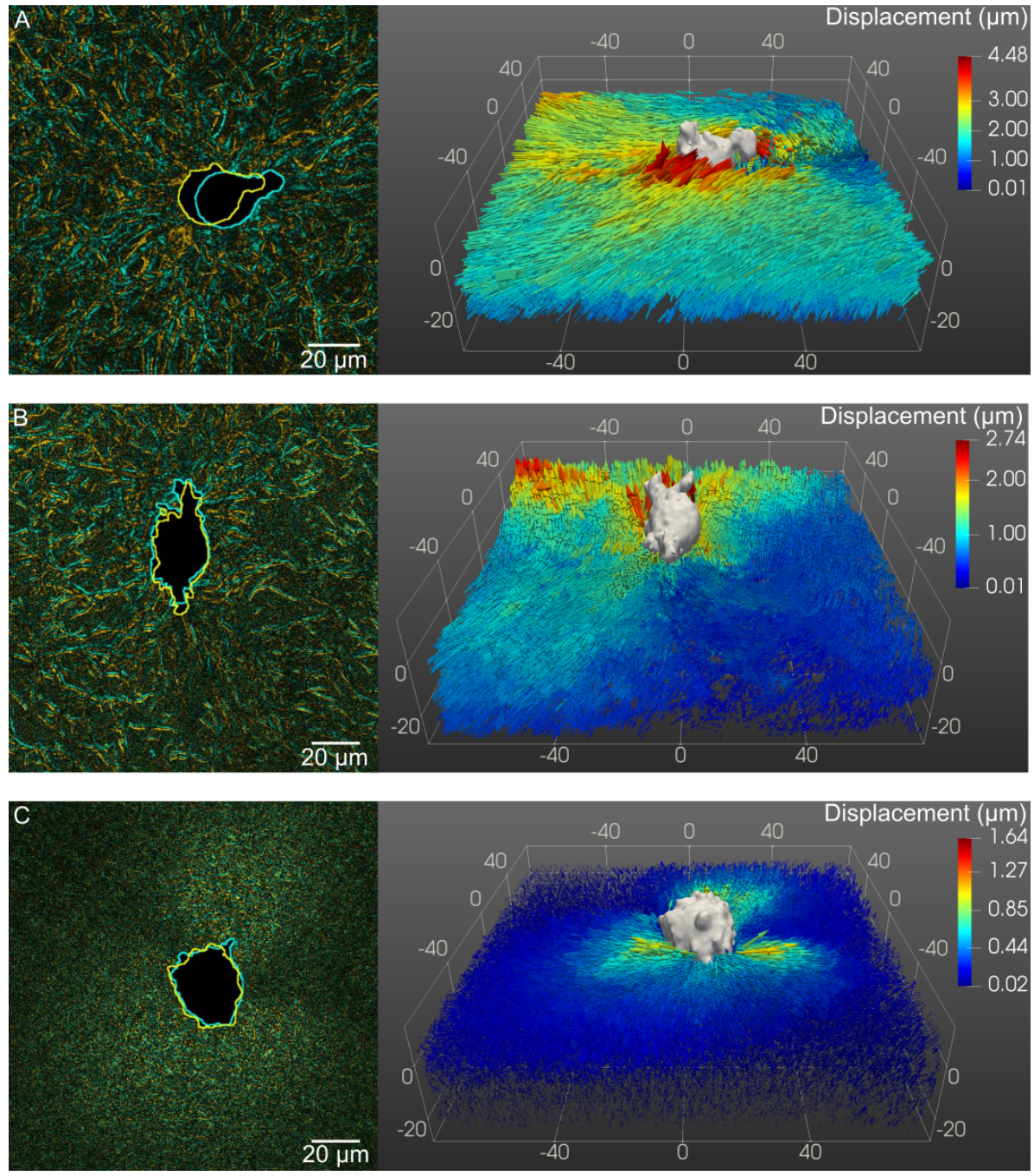

FIGURE 9 J82 cell migrating in three different gels (A) $0.95 \mathrm{mg} / \mathrm{mL}$, (B) $1.8 \mathrm{mg} / \mathrm{mL}$, (C) $4.5 \mathrm{mg} / \mathrm{mL}$, respectively from top to bottom. On the left are shown two successive cell contours (yellow is for time $t_{1}=0$ and cyan is at $t_{2}=10 \mathrm{~min}$ ). On the right are the results of the correlation algorithm showing 3D displacements in the whole field with the cell image (in grey) superposed. Displacements are much larger for the soft gel at the concentration of 0.95 $\mathrm{mg} / \mathrm{mL}$. Images were made using Paraview software [1]. 